Linear Filtering of the Sum of Two Known Stochastic Processes

Sayran Hmza Raheem

\title{
Linear Filtering of the Sum of Two Known Stochastic Processes
}

\author{
Sayran Hmza Raheem
}

University of Garmian - College of Languages and Human Science

\section{Received 24 April 2016 ; Accepted 19 September 2016}

\begin{abstract}
$\underline{\text { Abstract }}$
The linear filtering got the great attention of statisticians and applied mathematician; therefore the present study aims at finding the linear filtering of stationary stochastic process and that is when we know the values of the sum of two stochastic processes at all moments of the time and when $t \geq 0$, and this requires us to know the spectral density function $f_{X X}(\lambda)$ for the stochastic processes. In this paper, we opted to take two cases after giving the necessary definitions for all important terms and finding the spectral density function for each stochastic processes (Poisson process and Wide Sense Markov process) ; in the first case we supposed that both of the stochastic processes are stationary Poisson processes and after finding the linear filtering we compute the mean square filtering error ; and in second case we suppose one of the stochastic process is Poisson process and the other is wide sense Markov process also in this case we find the mean square filtering error.
\end{abstract}

Keywords: linear Filtering; Stochastic Process; Wide Sense; stationary Markov process 


\section{Linear Filtering of the Sum of Two Known Stochastic Processes}

Sayran Hmza Raheem

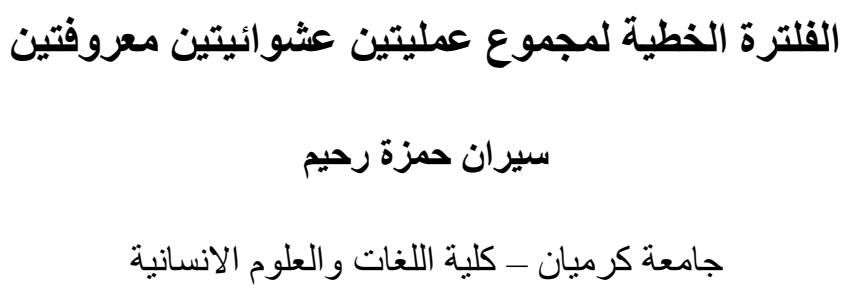

الخلاصة

نظر اللاهمية التي حضيت بها الفلترة الخطية للعمليات العشوائية لاى الاحصائيين وعلماء الرياضيات التطبيقية فان الهذف من هذا البحث هو الفلتره الخطية للعمليات العشوائية ، وهذا يتم بمعرفتنا لقيمة ناتج الجمع لعمليتين عثو ائيتين في كل لحظة من الزمن وفي حالة المستقبل و هذا تطلب منا حساب ومعرفة دالة الطيف لكل عملية عشو ائية واخترنا في هذا البحث ان نأخذ حالتين بعد اعطاء وتوضيح التعاريف الضروريه للمصطلحات امهمة التي ورد ذكر ها بالبحث و اعطاء قيمة دالة الطيف لكل عملية عشو ائية تم استخدامها :الحالة الاولى فرضنا ان كلتا العمليتن من عمليات بوايسون وبعد ايجاد دالة الفلترة الخطية قمنا بحساب قيمة مربع الخطأ للفلترة الخطية لضمان المزيد من المصداقية للنتائج وفي الحالة الثانية فرضنا احد العمليات العشو ائية احد انواع عمليات ماركوف والاخرى عملية بو ايسون وبعد جمعها وحساب الفلترة الخطية ايضا وكما الحالة الاولى حساب قيمة مربع الخطأ للفلترة الخطية الكلمات المفتاحية: الفلترة الخطية؛ العمليات العشو ائية؛ عمليات بو ايسون ؛ المعنى الواسع لعمليات ماركوف

\section{$\underline{\text { Introduction }}$}

Filtering of stochastic process has attracted a lot of attention. One of examples is target tracking, when the target is observed over a discrete time grid, corresponding to the successive passes of radar [5]. Any real data or signal measuring process includes some degree of noise from various possible sours. The desired signal may have added noise due to thermal or other physical effects related to the signal generation system, or it may be introduced noise due the measuring system or digital data sampling process. [11] the filtering problem in this paper is applied to two wellknown stochastic process (Poisson process and wide senses stationary Markova process) .we have to know that a filter is said to be linear if a set of it's inputs is linear and the filter operator is linear therefore we are dealing with linear filter operation. A linear filter is an operation that takes a function $X(t)$, the input and transform it into another function $X(t)-\varphi(t)$ the output, such that the transform of a linear combination of functions is equal to same linear combination of transforms of individual function of future values and for identification of interesting features 


\section{Linear Filtering of the Sum of Two Known Stochastic Processes}

Sayran Hmza Raheem

or removal of noise,[3]. It is fare to say that the spectral theory of stationary process is most useful in connection with linear filters so we have to find her the spectral representation of the stationary process to be able finding their filters since that the method of linear filtering is based on expression determining the spectral representation of the stochastic process ate the input and output of linear filtering Our work here should be interest to the applied mathematician. The paper Is organized as follows. In Section 2 we collect some important definitions for Markov process, Poisson process and Spectral density function. In Section 3 we find the spectral density function of wide sense Markov Process. In Section 4 we discuss our problem by taking two cases and we show that how can we find linear filtering of the sum of two known stochastic processes with the mean square filtering error for each case.

\section{Basic definitions}

\section{Wide sense Markov process}

The random process $\{X(t): t \in \tau\}$ is said to be a Markov process in the wide sense if $E\left\{|X(t)|^{2}\right\}<\infty$ and

$$
E\{X(t), X(1), \ldots, X(t-1)\}=E\{X(t) \mid X(t-1)\}
$$

\section{See [4]}

\section{2 .The Stochastic Poisson process}

A Poisson process with rate $(\lambda)$ is a counting process (a stochastic process) $\{N(t), t \geq 0\}$ for which

- $N(t) \geq 0$;

- The process has independent increments;

- The number of events in any interval of length $t$ the Poisson distribution with rate $(\lambda t)$ :

$$
P(N(s+t)-N(s)=n)=\frac{(\lambda t) e^{-(\lambda t)}}{n !}
$$




\section{Linear Filtering of the Sum of Two Known Stochastic Processes \\ Sayran Hmza Raheem}

$$
\text { for any } s \geq 0 \text { and } t>0
$$

[8] The first tow condition show the stationary of Poison process

The correlation function for Poisson process is

$$
B_{N N}(t)=\lambda t
$$

and the spectral density function for $B_{N N}(t)$ is

$f_{N N}=\frac{c}{\pi \lambda}, c=\frac{\pi(5+4 m)-2}{2}$ and $m$ is finite

\section{The spectral density function}

Every covariance function for stationary process has a Fourier transform (or rather an inverse Fourier transform) called the spectral density function [3];

i.e. if $B_{X X}(\tau)$ is the correlation function(cov.function) for the stochastic process $X(t)$ then the spectral density function of $X(t)$ can be represented in the following equation

$$
f_{X X}(\lambda)=\frac{1}{2 \pi} \int_{-\infty}^{\infty} e^{-i \lambda \tau} B_{X X}(\tau) \mathrm{d} \tau
$$

See [2]

\section{The spectral density function of wide sense Markov Process}

The auto-covariance function (the correlation function) of wide sense Markov process is exponential type

$$
\text { Let } \boldsymbol{B}_{X X}(\boldsymbol{\tau})=\sigma_{x}^{2} e^{-\alpha \tau} \quad \alpha>0, \sigma_{x}^{2}>0,0 \leq \tau<\infty \text { (3.1) }
$$

Which can easily be Fourier transformed to find the spectral density function [1]

So by using eq. (2.6) we get

$$
f_{X X}(\lambda)=\frac{1}{2 \pi} \int_{-\infty}^{\infty} e^{i \lambda \tau} \mathrm{m} e^{-\alpha|\tau|} \mathrm{d} \tau
$$




\section{Linear Filtering of the Sum of Two Known Stochastic Processes}

\section{Sayran Hmza Raheem}

$$
\begin{aligned}
& =\frac{1}{2 \pi} \int_{-\infty}^{\infty} e^{-\alpha|\tau|-i \lambda \tau} \mathrm{d} \tau \\
& =\frac{2 m}{2 \pi}\left\{\int_{-\infty}^{0} e^{(\alpha-i \lambda) \tau} d \tau+\int_{0}^{\infty} e^{-(\alpha+i \lambda) \tau} d \tau\right\} \\
& =\frac{2 m}{2 \pi}\left\{\frac{1}{\alpha-i \lambda}+\frac{1}{\alpha+i \lambda}\right\} \\
& =\frac{m}{2 \pi}\left\{\frac{\alpha+i \lambda+\alpha-i \lambda}{\alpha^{2}+\lambda^{2}}\right\}=\frac{m \alpha}{\pi\left(\alpha^{2}+\lambda^{2}\right)} \\
& =\frac{A}{\alpha^{2}+\lambda^{2}} \quad \text { where } A=\frac{m \alpha}{\pi}
\end{aligned}
$$

\section{The statement of the problem}

Let $\mathrm{X}(\mathrm{t})$ be a stochastic process and suppose that it is represent a "signal"(telephone message, radio broadcast, etc). But in our daily life and because of the existence of noise (which will also assume as a stochastic process $\mathrm{Y}(\mathrm{t}))$ that cams from the new technology or even our voice; $\mathrm{X}(\mathrm{t})$ will become spoiled and which is mean that we got a new function which came from the sum of these stochastic processes (signal and noise) :

$$
M(t)=X(t)+Y(t)
$$

In this paper we'll consider the problem of reconstruction of the value of a known stochastic process (noise) at the time $(t+\tau)$, where $\tau \geq 0$, we'll refer to the problem of linear filtering of stochastic process

\section{Linear Filtering of the Sum of Two stationary Poisson processes}

If both stochastic processes are stationary Poisson process(signal $N_{1}(t)$ and noise $N_{2}(t)$ ) we will consider the case where the value of the sum $N(t)=N_{1}(t)+N_{2}(t)$ (which is also Poisson process) are known at all moments of time, and by (2.4) the correlation function for $N_{1}(t) \&$ $N_{2}(t)$ that are

$$
B_{N_{1} N_{1}}(\tau)=\lambda \tau \quad \text { and } \quad B_{N_{2} N_{2}}(\tau)=\lambda \tau
$$

The signal and noise are not correlated with each other, i.e. 


\section{Linear Filtering of the Sum of Two Known Stochastic Processes}

Sayran Hmza Raheem

$$
\boldsymbol{E} N_{1}(t) \overline{N_{2}(s)}
$$

For any $\mathrm{t}$ and $\mathrm{s}$ the correlation function of stationary Poisson process fall of fast enough to have spectral density then because of (4.2)

$$
B_{N_{1} N_{1}}(\tau)=B_{N N}(\tau)-B_{N_{2} N_{2}}(\tau)
$$

and

$$
f_{N_{1} N_{1}}(\tau)=f_{N N}(\tau)-f_{N_{2} N_{2}}(\tau)
$$

Then $\quad \frac{c_{1}}{\pi \lambda}=f_{N N}-\frac{c_{2}}{\pi \tau}$ wher $c_{1}=\frac{\pi\left(5+4 m_{1}\right)-2}{2}$,

and $c_{2}=\frac{\pi\left(5+4 m_{2}\right)-2}{2}, m$ is constant.

So

$$
f_{N N}=\frac{c_{3}}{\pi \lambda} \quad \text { Where } c_{3}=c_{1}+c_{2}
$$

The linear filtering problem is equivalent to geometric problem of dropping a perpendicular from the point $N_{1}(t+\tau)$ of Hilbert space $H$ onto the linear subspace $H^{N}(t)$ spanned by the set of all vectors $N(t-s), s \geq 0$ [10] so we have to find the vector $L_{\tau}(t)$ in $H^{Z}(t)$ such that:

$$
\left(N_{1}(t+\tau)-L_{\tau}(t), N(t-s)\right)=0 \text { for } s \geq 0
$$

$L_{\tau}(t)$ satisfied this condition is uniquely defined therefore the random variable $L_{\tau}(t)$ is the best approximation to $\quad N_{1}(t+\tau)$ which depends linearly on $N(t-s), s \geq 0$ since $L_{\tau}(t)$ belong to $H^{N}(t)$ that means there is a sequence converges in the mean square to $L_{\tau}(t)$ and it can be written as the following:

$$
\begin{gathered}
\sum_{\mathrm{k}=1}^{\mathrm{n}_{1}} \alpha_{\mathrm{k}}^{(1)} \mathrm{N}\left(\mathrm{t}-\mathrm{s}_{\mathrm{k}}^{(1)}\right) \sum_{\mathrm{k}=1}^{\mathrm{n}_{2}} \alpha_{\mathrm{k}}^{(2)} \mathrm{N}\left(\mathrm{t}-\mathrm{s}_{\mathrm{k}}^{(2)}\right), \ldots \\
\ldots \sum_{\mathrm{k}=1}^{\mathrm{n}_{\mathrm{m}}} \alpha_{\mathrm{k}}^{(\mathrm{m})} \mathrm{N}\left(\mathrm{t}-\mathrm{s}_{\mathrm{k}}^{(\mathrm{m})}\right)
\end{gathered}
$$

Since we can write any formula of a stochastic process by another useful form in Fourier integral: 
Linear Filtering of the Sum of Two Known Stochastic Processes

Sayran Hmza Raheem

$$
X(t)=\int_{-\infty}^{\infty} e^{i \lambda \tau} d z(\lambda)
$$

and $z(\lambda)$ is a random function with uncorrelated increment satisfying the relation

$$
E|Z(\lambda+\Delta \lambda)-Z(\lambda)|^{2}=\int_{\lambda}^{\lambda+\Delta \lambda} f_{x x}(\lambda) d \lambda
$$

By the same way we can rewrite every element of the sequence (4.7) also we can write

$$
\begin{aligned}
\sum_{k=1}^{n_{m}} \alpha_{k}^{(m)} N\left(t-s_{k}^{(m)}\right)=\int_{-\infty}^{\infty} e^{i \lambda \tau} \Phi_{\tau, m}(\lambda) d Z_{Y}(\lambda) \quad \text { where } \Phi_{\tau, m} \\
=\sum_{k=1}^{n_{m}} a_{k}^{(m)} e^{-i s_{k}^{(m)} \lambda}
\end{aligned}
$$

So

$$
E\left|\sum_{k=1}^{n_{m}} \alpha_{k}^{(m)} N\left(t-s_{k}^{(m)}\right)-\sum_{k=1}^{n_{m+p}} \alpha_{k}^{(m+p)} N\left(t-s_{k}^{(m+p)}\right)\right|^{2}
$$

$=E\left|\int_{-\infty}^{\infty} e^{i \lambda \tau}\left[\Phi_{\tau, m}(\lambda)-\left[\Phi_{\tau, m+p}(\lambda)\right] d Z_{Y}(\lambda)\right]\right|^{2}$

$E\left[\int_{-\infty}^{\infty} e^{i \lambda \tau}\left[\Phi_{\tau, m}(\lambda)-\Phi_{\tau, m+p}(\lambda)\right] d Z_{Y}(\lambda)\right]$

$$
\begin{aligned}
& {\left[\overline{\left.\int_{-\infty}^{\infty} e^{\imath \lambda \tau}\left[\Phi_{\tau, m}(\lambda)-\Phi_{\tau, m+p}(\lambda)\right] d Z_{Y}(\lambda)\right]}\right.} \\
= & \int_{-\infty}^{\infty}\left|\Phi_{\tau}(\lambda)-\Phi_{\tau, m}(\lambda)\right|^{2} f_{N N}(\tau) d \lambda \\
= & \int_{-\infty}^{\infty}\left|\Phi_{\tau}(\lambda)-\Phi_{\tau, m}(\lambda)\right|^{2} \frac{c_{3}}{\pi \lambda} d \lambda=0 \text { where } c_{3}=c_{1}+c_{2}
\end{aligned}
$$

This implies that we can represent $L_{\tau}(t)$ as an integral of the form

$$
L_{\tau}(t)=\int_{-\infty}^{\infty} e^{i \lambda \tau} \Phi_{\tau}(\lambda) d Z_{Y}(\lambda)
$$

and $\quad \lim _{m \rightarrow \infty} \int_{-\infty}^{\infty}\left|\Phi_{\tau}(\lambda)-\Phi_{\tau, m}(\lambda)\right|^{2} f_{Z Z}(\lambda) d \lambda=0$ 


\section{Linear Filtering of the Sum of Two Known Stochastic Processes}

Sayran Hmza Raheem

$$
\begin{gathered}
\text { or } \lim _{m \rightarrow \infty} \int_{-\infty}^{\infty}\left|\Phi_{\tau}(\lambda)-\Phi_{\tau, m}(\lambda)\right|^{2} \frac{c_{3}}{\pi \lambda} d \lambda=0 \\
\lim _{m \rightarrow \infty} \frac{c_{3}}{\pi} \int_{-\infty}^{\infty} \frac{\left|\Phi_{\tau}(\lambda)-\Phi_{\tau, m}(\lambda)\right|^{2}}{\lambda} d \lambda=0
\end{gathered}
$$

We can rewrite the form (4.6) according to the formula

$$
\begin{aligned}
& N(t)=N_{1}(t)+N_{2}(t) \quad \text { and (4.2) as } \\
& \quad\left(N_{1}(t+\tau), N_{1}(t-s)-\left(L_{\tau}(t), N(t-s)\right)=0 \text { for } s \geq 0\right.
\end{aligned}
$$

In the previous equation we have a scalar product substitute (4.14) in to (4.17) and since

$$
\begin{gathered}
N(t-s)=N_{1}(t-s)+N_{2}(t-s) \text { So we can rewrite }(4.17) \text { as } \\
B_{X X}(\tau-s)-\Phi_{\tau}(\lambda) B_{Z}(t-s)=0 \\
\quad \text { or } \int_{-\infty}^{\infty} e^{i s \lambda}\left[e^{i \tau \lambda} f_{X X}(\lambda)-\Phi_{\tau}(\lambda) f_{Z Z}(\lambda)=0\right.
\end{gathered}
$$

Also we can obtain equation (4.19) by substituting (4.14) and the spectral representation of the processes $N_{1}(t)$ and $N(t)$ into (4.17) thus the solution of linear filtering problem reduces to finding the spectral characteristic for filtering $\Phi_{\tau}(\lambda)$ and the following conditions are sufficient for a function $\Phi_{\tau}(\lambda)$ to be the spectral characteristic for filtering [10]:

1. The function $\Phi_{\tau}(\lambda)$ is analytic in the lower half-plane, and as in $|\lambda| \rightarrow \infty$ in the lower half-plane, $\Phi_{\tau}(\lambda)$ grows no faster than some power of $|\lambda|$

2. The function $\Psi_{\tau}(\lambda)=e^{i \lambda \tau} f_{N_{1 N_{1}}}(\lambda)-\Phi_{\tau}(\lambda) f_{N N}(\lambda)$ is analytic in the upper half-plane, and as $|\lambda| \rightarrow \infty$ in the upper half-plane, $\Psi_{\tau}(\lambda)$ falls off faster than $|\lambda|^{-1-\varepsilon}$, where $\varepsilon>0$

3. The integral along the real axis of the function $\int_{-\infty}^{\infty}\left|\Phi_{\tau}\right|^{2} f_{N N}(\lambda) d \lambda$ is bounded. i.e., 


\section{Linear Filtering of the Sum of Two Known Stochastic Processes}

\section{Sayran Hmza Raheem}

$$
\int_{-\infty}^{\infty}\left|\Phi_{\tau}\right|^{2} f_{N N}(\lambda) d \lambda<\infty
$$

Now since we said in our assumption that we know the value of the sum of the noise and signal at all the moments of time so in this case, the spectral characteristic function $\Phi_{\tau}(\lambda)$ for filtering must satisfy the condition

$$
\int_{-\infty}^{\infty} e^{i s \lambda}\left[f_{N_{1} N_{1}}(\lambda)-\Phi_{\tau}(\lambda) f_{N N}(\lambda)\right]=0 \text { for }-\infty \leq s \leq \infty(4.21)
$$

It is clear that eq. (4.21) is a Fourier transform and since the function $\left[f_{N_{1} N_{1}}(\lambda)-\Phi_{\tau}(\lambda) f_{N N}(\lambda)\right]$ is an absolutely integral function on $\mathbb{R}$ whose Fourier transform is identically equal to zero then [9]

$$
\begin{gathered}
f_{N_{1} N_{1}}(\lambda)-\Phi_{\tau}(\lambda) f_{N N}(\lambda)=0 \\
\Phi_{\tau}(\lambda)=\frac{f_{N_{1} N_{1}}(\lambda)}{f_{N_{2} N_{2}}(\lambda)}=\frac{c_{1} / \pi \lambda}{c_{2} / \pi \lambda} \\
\Phi_{\tau}(\lambda)=\frac{c_{1}}{c_{2}}, \quad c_{1}=\frac{\pi\left(5+4 m_{1}\right)-2}{2}, c_{2}=\frac{\pi\left(5+4 m_{2}\right)-2}{2}
\end{gathered}
$$

We have to find now the mean square filtering error, since that the random variable $L_{\tau}(t)$ is the best approximation to the signal $N_{1}(t+\tau)$ which depends linearly on the $N(t-s), s \geq 0$ so:

$$
\sigma_{\tau}^{2}=E\left|N_{1}(t+\tau)-L_{\tau}(t)\right|^{2}
$$

And the difference $X(t+\tau)-L_{\tau}(t)$ is orthogonal to the vector $L_{\tau}(t)$, since $L_{\tau}(t)$ belong to Hilbert subspace $H^{Z}(t)$ we find that [10]:

$$
\begin{aligned}
& \sigma_{\tau}^{2}=E\left|N_{1}(t+\tau)-L_{\tau}(t)\right| \overline{X(t+\tau)} \\
& =E\left|N_{1}(t+\tau)\right|^{2}-E L_{\tau}(t) \overline{\left[\left(X(t+\tau)-L_{\tau}(t)+L_{\tau}(t)\right]\right.} \\
& =E\left|N_{1}(t+\tau)\right|^{2}-E\left|L_{\tau}(t)\right|^{2} \\
& =E\left(N_{1}(t+\tau) \overline{\left.N_{1}(t+\tau)\right)}-E\left|L_{\tau}(t)\right|^{2}\right.
\end{aligned}
$$


Linear Filtering of the Sum of Two Known Stochastic Processes

Sayran Hmza Raheem

$$
\begin{aligned}
& =B_{N_{1} N_{1}}((t+\tau)-(t+\tau))-E\left|L_{\tau}(t)\right|^{2} \\
& =B_{N_{1 N_{1}}}(0)-E\left|L_{\tau}(t)^{2}\right| \\
& =\int_{-\infty}^{\infty} f_{N_{1 N_{1}}}(\lambda) d \lambda-\int_{-\infty}^{\infty}\left|\Phi_{\tau}\right|^{2} f_{N N}(\lambda) d \lambda \\
& =\int_{-\infty}^{\infty} f_{N N_{1}}(\lambda) d \lambda-\int_{-\infty}^{\infty}\left(\frac{f_{N_{1 N_{1}}}(\lambda)}{f_{N N}(\lambda)}\right)^{2} f_{N N} d \lambda \\
& =\int_{-\infty}^{\infty} f_{N_{1} N_{1}}(\lambda) d \lambda-\int_{-\infty}^{\infty} \frac{\left(f_{N_{1} N_{1}}(\lambda)\right)^{2}}{f_{N N}(\lambda)} d \lambda \\
& =\int_{-\infty}^{\infty} f_{N_{1 N_{1}}}(\lambda) d \lambda-\int_{-\infty}^{\infty} \frac{\left(f_{N_{1} N_{1}}(\lambda)\right)^{2}}{f_{N_{1} N_{1}}(\lambda)+f_{N_{2} N_{2}(\lambda)}} d \lambda \\
& =\int_{-\infty}^{\infty} \frac{f_{N_{1} N_{1}}(\lambda) f_{N_{2} N_{2}(\lambda)}}{f_{N_{1 N_{1}}}(\lambda)+f_{N_{2} N_{2}(\lambda)}} d \lambda \\
& =\int_{-\infty}^{\infty} \frac{c_{1} / \pi \lambda \cdot{ }^{c_{2}} / \pi \lambda}{c_{1} / \pi \lambda+{ }^{c_{2}} / \pi \lambda} d \lambda \\
& =\int_{-\infty}^{\infty} \frac{c_{1} c_{2} /(\pi \lambda)^{2}}{c_{3} / \pi \lambda} d \lambda \quad \text {, where } c_{3}=c_{1}+c_{2} \\
& =\frac{c_{1} c_{2}}{c_{3} \pi} \int_{-\infty}^{\infty} \frac{1}{\lambda} d \lambda \\
& \sigma_{\tau}^{2}=\frac{c_{1} c_{2}}{c_{3} \pi} \ln \lambda
\end{aligned}
$$

This shows that complete suppression of the noise is possible only when the spectra of the signal and the noise do not overlap [10]

\section{Linear Filtering of the Sum of wide sense Markov Process and Poisson process}

- We'll consider the case where the signal $\{X(t) ; t \geq 0\}$ is stationary process of Markov type (wide sense Markov process) and the noise $N(t) ; t \geq 0\}$ is Poisson process then by(5)\&(3) 


$$
\begin{gathered}
f_{X X}(\lambda)=\frac{A}{\lambda^{2}+\alpha^{2}}, \quad f_{N N}(\lambda)=\frac{c}{\pi \lambda} \\
K(t)=X(t)+N(t) \\
f_{X X}(\tau)=f_{N N}(\tau)-f_{K K}(\tau) \\
f_{K K}(\lambda)=\frac{A}{\lambda^{2}+\alpha^{2}}+\frac{c}{\pi \lambda}=\frac{A \pi \lambda+C\left(\lambda^{2}+\alpha^{2}\right)}{\pi \lambda\left(\lambda^{2}+\alpha^{2}\right)}
\end{gathered}
$$

According to condition (b), the function

$$
\begin{aligned}
& \Psi_{\tau}(\lambda)=e^{i \lambda \tau} f_{X X}(\lambda)-\Phi_{\tau}(\lambda) f_{Z Z}(\lambda) \\
& e^{i \lambda \tau} \frac{A}{\lambda^{2}+\alpha^{2}}+\Phi_{\tau}(\lambda) \frac{A \pi \lambda+C\left(\lambda^{2}+\alpha^{2}\right)}{\pi \lambda\left(\lambda^{2}+\alpha^{2}\right)} \\
& \frac{A \pi \lambda e^{i \lambda \tau}-\Phi_{\tau}(\lambda)\left[A \pi \lambda+C\left(\lambda^{2}+\alpha^{2}\right)\right]}{\pi \lambda\left(\lambda^{2}+\alpha^{2}\right)}
\end{aligned}
$$

must be analytic in the upper half-plane. This implies that the zeros of the denominator of $\Psi_{\tau}(\lambda)$ at the point $\lambda=0$ and $\lambda=i \alpha$ must be canceled by zeros of the numerator at the same points, so that

$$
\begin{aligned}
& \Phi_{\tau}(i \alpha)=\frac{A i \alpha \pi e^{-\alpha \tau}}{A \pi i \alpha} \\
& \Phi_{\tau}(i \alpha)=e^{-\alpha \tau}, \quad \Phi_{\tau}(0)=0
\end{aligned}
$$

Since the function $\Phi_{\tau}(\lambda)$ must be analytic in the lower half-plane and the function $\Psi_{\tau}(\lambda)$ must be analytic in the upper half-plane it follows that $\Psi_{\tau}(\lambda)$ and $\Phi_{\tau}(\lambda)$ can have no singularities in common and because of the form (4.33)this implies that $\Phi_{\tau}(\lambda)$ can have no singularities in any point so that

$$
\Phi_{\tau}(\lambda)=e^{i \lambda \tau}
$$

We have to find now the mean square filtering error by using eq. (4.27) 
Linear Filtering of the Sum of Two Known Stochastic Processes

Sayran Hmza Raheem

$$
\sigma_{\tau}^{2}=\int_{-\infty}^{\infty} f_{X X}(\lambda) d \lambda-\int_{-\infty}^{\infty}\left|\Phi_{\tau}\right|^{2} f_{K K}(\lambda) d \lambda
$$

Substitute (3.3) and (2.5) in (4.27)

$$
\begin{gathered}
=\int_{-\infty}^{\infty} \frac{A}{\lambda^{2}+\alpha^{2}} d \lambda-\int_{-\infty}^{\infty}\left|e^{i \lambda \tau}\right|^{2} \frac{A \pi \lambda+C\left(\lambda^{2}+\alpha^{2}\right)}{\pi \lambda\left(\lambda^{2}+\alpha^{2}\right)} d \lambda \\
=A \int_{-\infty}^{\infty} \frac{d \lambda}{\lambda^{2}+\alpha^{2}} d \lambda-\int_{-\infty}^{\infty} e^{2 i \lambda \tau} \frac{A \pi \lambda+C\left(\lambda^{2}+\alpha^{2}\right)}{\pi \lambda\left(\lambda^{2}+\alpha^{2}\right)} d \lambda \\
=A \frac{1}{\alpha} \tan ^{-1}\left(\frac{\lambda}{\alpha}\right)-\int_{-\infty}^{\infty} e^{2 i \lambda \tau}\left(\frac{A}{\left(\lambda^{2}+\alpha^{2}\right)}+\frac{c}{\pi \lambda}\right) d \lambda
\end{gathered}
$$

then

$$
\left.\int_{-\infty}^{\infty} \mathrm{e}^{2 \mathrm{i} \lambda \tau}\left(\frac{\mathrm{A}}{\left(\lambda^{2}+\alpha^{2}\right)}+\frac{\mathrm{c}}{\pi \lambda}\right) \mathrm{d} \lambda=\mathrm{A} \int_{-\infty}^{\infty} \frac{\mathrm{e}^{2 \mathrm{i} \lambda \tau}}{\left(\lambda^{2}+\alpha^{2}\right)} \mathrm{d} \lambda+\frac{\mathrm{c}}{\pi} \int_{-\infty}^{\infty} \frac{\mathrm{e}^{2 \mathrm{i} \lambda \tau}}{\lambda}\right) \mathrm{d} \lambda
$$

To find $\int_{-\infty}^{\infty} \frac{e^{2 i \lambda \tau}}{\left(\lambda^{2}+\alpha^{2}\right)} d \lambda$

Let

$u=e^{2 i \lambda \tau}, d u=2 i \tau e^{2 i \lambda \tau} d \lambda$

$d v=\int_{-\infty}^{\infty} \frac{d \lambda}{\left(\lambda^{2}+\alpha^{2}\right)} \quad, v=\frac{1}{\alpha} \tan ^{-1}\left(\frac{\lambda}{\alpha}\right)$

So $\int_{-\infty}^{\infty} \frac{e^{2 i \lambda \tau}}{\left(\lambda^{2}+\alpha^{2}\right)} d \lambda$

$=e^{2 i \lambda \tau}\left(\frac{1}{\alpha} \tan ^{-1}\left(\frac{\lambda}{\alpha}\right)\right)-2 i \tau \int_{-\infty}^{\infty}\left(\frac{1}{\alpha} \tan ^{-1}\left(\frac{\lambda}{\alpha}\right)\right) e^{2 i \lambda \tau} d \lambda$

$=e^{2 i \lambda \tau}\left(\frac{1}{\alpha} \tan ^{-1}\left(\frac{\lambda}{\alpha}\right)\right)+\frac{1}{2 \alpha} i\left(-e^{2 \alpha \tau} \operatorname{Ei}\left(2 \tau(\alpha+i \lambda)+\quad e^{2 i \tau \lambda} \tan ^{-1}\left(\frac{\lambda}{\alpha}\right)\right)\right.$

(4.39)

To find $\left.\int_{-\infty}^{\infty} \frac{e^{2 i \lambda \tau}}{\lambda}\right) d \lambda$ 


\section{Linear Filtering of the Sum of Two Known Stochastic Processes}

\section{Sayran Hmza Raheem}

$\left.\int_{-\infty}^{\infty} \frac{e^{2 i \lambda \tau}}{\lambda}\right) d \lambda=E \mathrm{i}(2 i \lambda \tau)$

Substitute (4.39) and (4.40) in (4.38)

$\int_{-\infty}^{\infty} e^{2 i \lambda \tau}\left(\frac{A}{\left(\lambda^{2}+\alpha^{2}\right)}+\frac{c}{\pi \lambda}\right) d \lambda=$

$A e^{2 i \lambda \tau}\left(\frac{1}{\alpha} \tan ^{-1}\left(\frac{\lambda}{\alpha}\right)\right)+\frac{1}{2 \alpha} i\left(-e^{2 \alpha \tau} E i(2 \tau(\alpha+i \lambda)+\right.$

$\left.e^{2 i \tau \lambda} \tan ^{-1}\left(\frac{\lambda}{\alpha}\right)\right)+$

$\frac{c}{\pi} E \mathrm{i}(2 i \lambda \tau)$

Substitute (4.41) in (4.27)

$$
\begin{array}{r}
\sigma_{\tau}^{2}=A \frac{1}{\alpha} \tan ^{-1}\left(\frac{\lambda}{\alpha}\right)-A e^{2 i \lambda \tau}\left(\frac{1}{\alpha} \tan ^{-1}\left(\frac{\lambda}{\alpha}\right)\right)+ \\
\frac{1}{2 \alpha} i\left(-e^{2 \alpha \tau} E i\left(2 \tau(\alpha+i \lambda)+\tan ^{-1}\left(\frac{\lambda}{\alpha}\right)\right)+\frac{c}{\pi} E i(2 i \lambda \tau)\right.
\end{array}
$$

Where Ei is called an exponential integral and The standard definition of the exponential integral is [6]

$$
E_{n}(x)=\int_{1}^{\infty} \frac{e^{-x t}}{t^{n}} d t \quad x>o, n=0,1, . .
$$

It is also defined by the principal value of the integral

$$
E i=-\int_{-x}^{\infty} \frac{e^{-t}}{t} d t=\int_{-\infty}^{x} \frac{e^{t}}{t} d t
$$

We see that the mean square filtering error takes its minimum values 


\section{Linear Filtering of the Sum of Two Known Stochastic Processes \\ Sayran Hmza Raheem}

\section{Conclusion and Numerical Results}

From our present study of the filtering problem for a known stationary process and finding the mean square filtering error the following could be seen

- In order to find the linear filtering of any stationary stochastic process we have to find first the spectral density function for the process and for Wide sense Markov process

$$
\left(f_{X X}(\lambda)=\frac{A}{\alpha^{2}+\lambda^{2}} \quad \text { where } A=\frac{m \alpha}{\pi}, m \text { con. }\right)
$$

And the spectral density function for the sum of stationary Poisson process and wide sense Markov process is:

$$
f_{K K}(\lambda)=\frac{A \pi \lambda+C\left(\lambda^{2}+\alpha^{2}\right)}{\pi \lambda\left(\lambda^{2}+\alpha^{2}\right)}, \text { wher } A \& C \text { are con }
$$

- The solution of linear filtering problem reduces to finding the spectral characteristic for filtering $\Phi_{\tau}(\lambda)$ in two cases :

In the first case when both of noise and signal are stationary Poisson process

$$
\begin{gathered}
\Phi_{\tau}(\lambda)==\frac{\boldsymbol{c}_{\mathbf{1}}}{\boldsymbol{c}_{\mathbf{2}}}, \quad \mathrm{c}_{1}=\frac{\pi\left(5+4 m_{1}\right)-2}{2}, c_{2}=\frac{\pi\left(5+4 m_{2}\right)-2}{2}, \\
\text { where } m_{1}, m_{2} \text { are finit }
\end{gathered}
$$

and the second case when the noise assumed to be wide sense Markov process and we take the time $t \geq o$

$$
\Phi_{\tau}(\lambda)=e^{i \lambda \tau}
$$




\section{Linear Filtering of the Sum of Two Known Stochastic Processes \\ Sayran Hmza Raheem}

- After finding the value of $\Phi_{\tau}(\lambda)$ it was necessary for our study to find the mean square filtering error, which we find that In the first case when both of noise and signal are stationary Poisson process $\sigma_{\tau}^{2}=\frac{c_{1} c_{2}}{c_{3} \pi} \ln \lambda$

And in the second case

$$
\begin{aligned}
& \sigma_{\tau}^{2}=A \frac{1}{\alpha} \tan ^{-1}\left(\frac{\lambda}{\alpha}\right)-A e^{2 i \lambda \tau}\left(\frac{1}{\alpha} \tan ^{-1}\left(\frac{\lambda}{\alpha}\right)\right)+\quad \frac{1}{2 \alpha} i\left(-e^{2 \alpha \tau} E i(2 \tau(\alpha+i \lambda)+\right. \\
& \left.e^{2 i \tau \lambda} \tan ^{-1}\left(\frac{\lambda}{\alpha}\right)\right)+\quad \frac{c}{\pi} E \mathrm{i}(2 i \lambda \tau)
\end{aligned}
$$

\section{$\underline{\text { References }}$}

1. Bucher,Ch.,"Computational Analysis of Randomness in Structural Mechanics: Structures and Mechanics", CRC Press/Balkema,London,2009;pp84

2. Grigoriu, M. ,"Stochastic Calculus Application in Science and Engineering" Birkhaoser Boston,USA,(2002) ;pp133

3. Lindgreen, G., Rootzen, .H, Sandsten, M.," Stationary Stochastic Process for Scientists and Engineers" CRC Press London,(2014); pp133-82

4. Los ,C., "Financial Market Risk : 'Measurement and Analysis " Routledge, New Romany, (2003); pp 39

5. Makhanin, O.," Filtering and Parameter Estimation for A jump Stochastic Process with Discrete Observations" New Mexico, (2008); pp1

6. Press,W.H, Teukolsky,S.A,Vetterling,W.T, Flannery,B.P "Numerical Recipes in $\mathrm{C}$, The Art of Scientific Computing" CAMBRIDG,New York, 1992;pp222 
Linear Filtering of the Sum of Two Known Stochastic Processes

\section{Sayran Hmza Raheem}

7. Rahiem, S., H., " Linear Extrapolation of Homogenous Poisson Process"M.sc. Thesis, College of Ibn Al-Haitham, university of Baghdad, Iraq, 2007; pp19-25

8. Ravindran, A.R.," Operations Research Methodologies", Taylor \&Francis group, New York, (2009); pp8-9.

9. Ruch, j., "Fourier Series, Integrals, Sampling From Basic Complex Analysis"; pdf

10. Yaglom, A.,M., "An Introduction to The Theory of Stationary Random Functions" Mineola, New York, (1962); pp168-170-169

11. Zaknich, A., "Principles of Adoptive Filters and Self-Learning Systems" Germany,2005;pp 3 